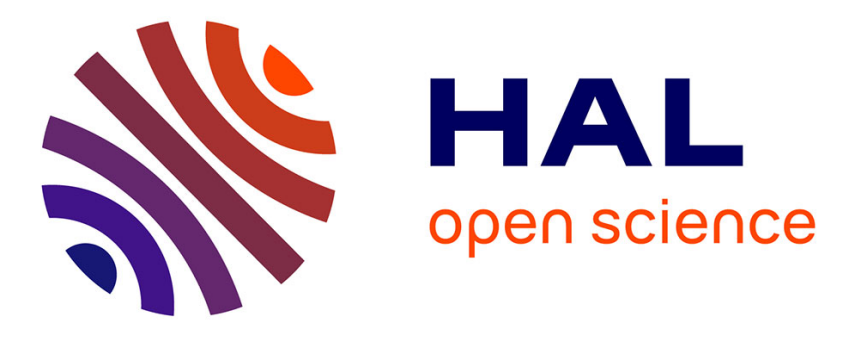

\title{
Experimental Localization Application in Opportunistic Scenario
}

Francesco Zorzi, Andrea Bardella, Tanguy Pérennou, Guodong Kang, Andrea Zanella

\section{- To cite this version:}

Francesco Zorzi, Andrea Bardella, Tanguy Pérennou, Guodong Kang, Andrea Zanella. Experimental Localization Application in Opportunistic Scenario. The Second International Workshop on Mobile Opportunistic Networking (ACM/SIGMOBILE MobiOpp 2010), Feb 2010, Pisa, Italy. 3p. hal00464302

\section{HAL Id: hal-00464302 https://hal.science/hal-00464302}

Submitted on 16 Mar 2010

HAL is a multi-disciplinary open access archive for the deposit and dissemination of scientific research documents, whether they are published or not. The documents may come from teaching and research institutions in France or abroad, or from public or private research centers.
L'archive ouverte pluridisciplinaire HAL, est destinée au dépôt et à la diffusion de documents scientifiques de niveau recherche, publiés ou non, émanant des établissements d'enseignement et de recherche français ou étrangers, des laboratoires publics ou privés. 


\title{
Experimental Localization Application in Opportunistic Scenario
}

\author{
Francesco Zorzi ${ }^{\dagger}$, Andrea Bardella ${ }^{\dagger}$, Tanguy Pérennou*, \\ Guodong Kang** and Andrea Zanella ${ }^{\dagger}$ \\ † Dipartimento di Ingegneria dell'Informazione, Università degli Studi di Padova, Italy \\ * ISAE, Université de Toulouse, France \\ ¥ Northwestern Polytechnical University, Xi'an, China \\ E-mail: \{zorzifra, bardella, zanella\}@dei.unipd.it, \{perennou, gkang\}@isae.fr
}

\begin{abstract}
Opportunistic localization is a new approach to the selflocalization problem that is recognized as one of the most critical for mobile users, in particular in indoor environments. The basic idea consists in allowing mobile users to exchange location information when they happen to be in radio range and to exploit this information in order to improve the self-localization accuracy of mobile users. This demo is aimed at proving the effectiveness of the opportunistic approach and identifying possible drawbacks and technical issues. To this end, we will realize a simple though realistic network deployment, where a mobile user, which performs a very basic min-max self localization procedure, tries to improve the accuracy of its location by communicating on an opportunistic basis with other nodes in spatial proximity. The demo will allow us to appreciate the actual benefit that the opportunistic paradigm brings to the self-localiztion problem and to compare the performance of different opportunistic localization algorithms.
\end{abstract}

\section{Categories and Subject Descriptors}

C.2.2 [Computer-Communication Networks]: Network Protocols

\section{General Terms}

Experimentation, Performance

\section{Keywords}

Localization, Testbed, Wireless Sensor Networks, Opportunistic Networks

\section{INTRODUCTION}

In this demo, we want to provide localization service for devices that do not adopt dedicated equipments. Achieving an accurate location estimation is difficult when considering

Permission to make digital or hard copies of all or part of this work for personal or classroom use is granted without fee provided that copies are not made or distributed for profit or commercial advantage and that copies bear this notice and the full citation on the first page. To copy otherwise, to republish, to post on servers or to redistribute to lists, requires prior specific permission and/or a fee.

MobiOpp '10, February 22-23, 2010, Pisa, Italy.

Copyright 2010 ACM 978-1-60558-925-1/10/02 ...\$10.00. cheap off-the-shelf mobile devices, particularly in indoors or urban environments. However, those nodes always have an inherent RF communication capability that can be used to communicate with other nodes and that always provide a Received Signal Strength (RSS) indicator. Thus we envision a scenario in which heterogeneous mobile nodes may have different localization accuracies obtained using information received from beacon nodes or exploiting other techniques. Moreover these nodes can communicate among themselves and exchange localization information in an opportunistic fashion, i.e. when in coverage range, and use it to update their position estimate. We want to show that these interactions among nodes can improve the localization accuracy under certain assumptions, as stated in [7,6]. Therefore we want to compare the localization error enabling or not the opportunistic scheme. In our deployment, we will use a number of beacon nodes with known positions and channel parameters as localization infrastructure. For the sake of reducing demo complexity, some fixed nodes with variable accuracy will be deployed to emulate the opportunistic nodes that provide opportunistic interactions with the mobile nodes carried by visitors of the demo. We will adopt both range-based and range-free algorithms, in order to take into account the availability of channel parameters. In the first case, the mobile node localizes itself using the anchor nodes informations and then opportunistically it will try to ameliorate its position estimation exchanging information with the opportunistic nodes, leveraging on ranging information. If anchor nodes do not provide such parameters, the mobile node will use a range free localization technique exploiting the only position information of anchor and opportunistic nodes, at most using the RSS samples as a proximity coefficient.

\section{OPPORTUNISTIC LOCALIZATION AL- GORITHMS}

In this demo, we will demonstrate the effectiveness of the localization results presented in $[5,6,7]$ also in a real-time implementation using TmoteSky sensor nodes.

The two papers tackle the problem of opportunistic localization in two different ways.

In [7], we assumed that nodes have a 2-D Gaussian distribution of native localization error, with zero mean and a certain variance $\sigma^{2}$, that corresponds to a mean error of $\sigma \sqrt{\frac{\pi}{2}}$. The mobile node performs the opportunistic update after a contact with an opportunistic node that is assumed to have a better self-localization capability. To better ap- 
preciate the effect of opportunistic localization, we defined the opportunistic gain metric $\Delta_{i}, i=A, B$, as

$$
\Delta_{i}=\frac{\sigma_{i} \sqrt{\pi / 2}-\tilde{\epsilon}}{\sigma_{i} \sqrt{\pi / 2}}
$$

where $\tilde{\epsilon}$ is the mean localization error after the opportunistic localization, whereas $\sigma_{i} \sqrt{\pi / 2}$ is the mean localization error of the node obtained by using the native localization scheme. Therefore, $\Delta$ represents the relative gain in the localization error obtained by using the opportunistic scheme. Fig. 1 shows the performance of ML algorithm [4] but, due to its complexity, is not very suitable for tiny devices like TmoteSky. Therefore we also proposed an heuristic algorithm, very light to implement and, under certain assumptions, also effective. In this solution, we consider that the opportunistic node has only one contact with other nodes.

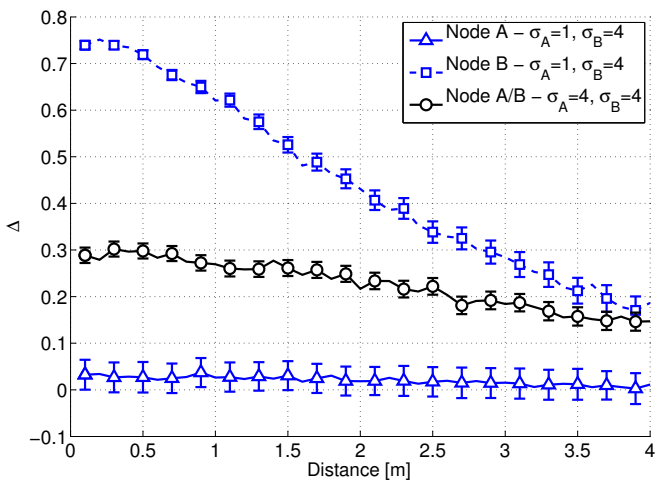

Figure 1: Relative localization error gain after an opportunistic update varying the hit distance

In [6] a different scenario is presented. A node, completely unaware of its position, collects several information from opportunistic nodes about localization. This information is then added into a matrix to create a Linear Matrix Inequality system [2]. The solution of this system is chosen as the final position estimation of the node. Also the Centroid algorithm can be used in some scenario to lighten the computational effort.

In this case, many contacts are needed to perform good localization results as shown in Fig. 2. Moreover, the information has to be obtained with the node always in the same position.

This scheme can be used both with range-free and rangebased information.

Therefore it is interesting to use a hybrid approach that takes into account the number of contacts of the mobile nodes. When only one or two opportunistic nodes reply to its request, then the node performs the ML algorithm beacuse it is not possible to have a meaningful solution for the LMI or Centroid algorithm. On the contrary this solution is preferred when many communications have been collected.

\section{DEMO SETUP}

The demo will be performed in an area where several sensor nodes will be deployed as shown in Fig. 3.

We will consider three different classes of nodes. In the first one we have beacon nodes, that know their position

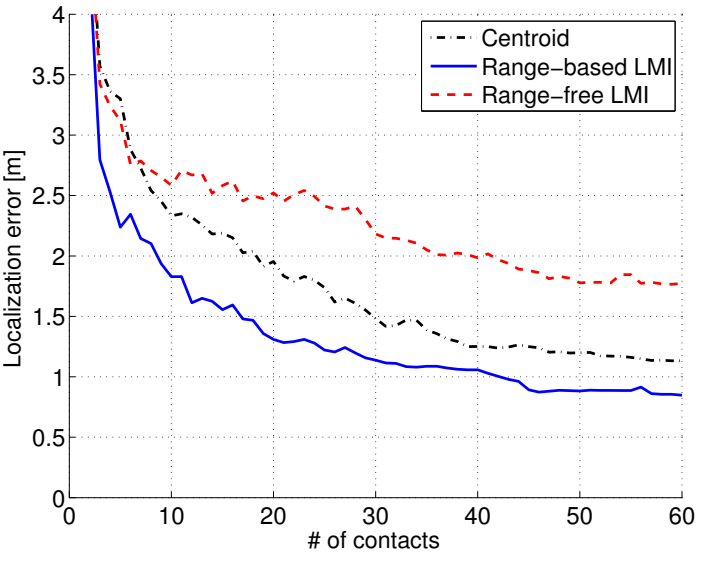

Figure 2: Localization performance of range-free LMI, range-based LMI and centroid algorithms, with $\sigma_{\psi}=2 \mathrm{~dB}$ and $\sigma_{l o c}=0 \mathrm{~m}$.

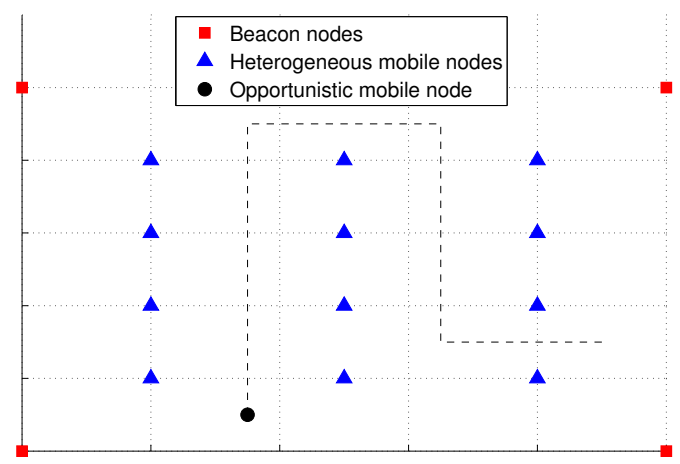

Figure 3: Nodes' deployment

and act like reference points for other nodes. In the second there are opportunistic nodes, that emulate mobile nodes and can be set with different positioning estimation accuracy to better understand the gain that we can achieve using opportunistic interactions in different scenarios. Finally, the mobile nodes, that will be carried by the visitors and that will be the target of our demonstration for localization estimation accuracy. The entire information exchanged by the mobile nodes will be processed by itself and sent to a laptot only to visualize the estimations on the screen. Moreover, we will use a pre-planned path that the opportunistic node will follow, in order to know the real position of the node while it is moving, using this information to characterize the positioning error.

In our experiments we used Tmote Sky sensor nodes [1] which are equipped with the Chipcon wireless transceiver CC2420 based on IEEE 802.15.4 standard (PHY and MAC layers). CC2420 operates in $2.4 \mathrm{GHz}$ ISM band at 250 kbps, uses DSSS modulation (the encoding scheme encodes 32 chips for a symbol of 4 bits and this encoded data is then OQPSK modulated) that provides the RSSI (Received Signal Strength Indicator).

We suppose that beacon nodes broadcast every $T_{b}$ seconds their position and the radio channel parameters, if available, 
in a coordinated way. The mobile node turns on and waits for the beacon messages in order to localize itself using MinMax algorithm [3]. After this phase, the mobile node waits a random time $T_{w}$ and then starts a scan-phase for opportunistic interactions. In the scan-phase the node transmits a request message periodically, with period $T$, to solicit potential neighbor nodes for opportunistic contacts. After each transmission, the node waits for a reply or for messages sent by other nodes for a fraction of time $\delta T$. The scan-phase lasts at most $N \times T$ seconds, where $N$ is a design parameter that represents the maximum number of opportunistic updatings between two consecutive position estimations using only the beacon nodes' information.

The message broadcasted by opportunistic nodes carry information concerning the current estimated node's position, the time since the last update with self-localization methods, the time since the last opportunistic update, the class of localization accuracy the node belongs to. Furthermore, the receiver extracts from the radio signal the Radio Signal Strength Indicator (RSSI) measure, which is then used by the opportunistic localization algorithms described above.

\section{CONCLUSIONS AND POSSIBLE EXTENSIONS}

The demo shows that opportunistic localization paradigm is effective in enhancing the node's localization accuracy in indoor environments, though the performance increment is strongly dependent on the node's mobility patterns, the heterogeneity of the opportunistic nodes and the accuracy of the ranging. Furthermore, we prove that some of the opportunistic algorithms proposed in $[5,6,7]$ are actually portable on low-end radio devices, such as Tmote-SKY sensor nodes, thus providing an effective way to enhance the localization capability of such nodes without requiring ancillary hardware. As a possible next step, we wish to investigate the potential benefits of applying tracking techniques, such as particle filters, in conjunction with the opportunistic position update schemes.

\section{ACKNOWLEDGMENTS}

This work was partly supported by the European Commission in the framework of the FP7 Network of Excellence in Wireless COMmunications NEWCOM++ (contract n. 216715), by French ANR Telecoms Project FIL and by "Fondazione Cassa di Risparmio Padova e Rovigo" under the project "A large scale wireless sensor network for pervasive city-wide ambient intelligence."

\section{REFERENCES}

[1] Tmote sky datasheet.

[2] L. Doherty, L. E. Ghaoui, and K. S. J. Pister. Convex position estimation in wireless sensor networks. In Proc. of IEEE INFOCOM, pages 1655-1663, Anchorage, AK, USA, April 2001.

[3] K. Langendoen and N. Reijers. Distributed localization in wireless sensor networks: a quantitative comparison. Comput. Networks, 43(4):499-518, 2003.

[4] N. Patwari, R. O'Dea, and Y. Wang. Relative location in wireless networks. In Proc. of the IEEE VTS 53rd Vehicular Technology Conference (VTC 2001 Spring), pages 1149-1153, Rhodes, Greece, May 2001.
[5] F. Zorzi, A. Bardella, T. PÃl'rennou, G. Kang, and A. Zanella. Analysis of opportunistic localization algorithms based on the linear matrix inequality method. In accepted as poster at Mobiopp 2010, Pisa, Italy, 2010.

[6] F. Zorzi, G. Kang, T. PÂl'rennou, and A. Zanella. Opportunistic localization scheme based on linear matrix inequality. In Proc. of WISP 2009, Budapest, Hungary, August 2009.

[7] F. Zorzi and A. Zanella. Opportunistic localization: modeling and analysis. In Proc. of the IEEE 69th Vehicular Technology Conference (VTC Spring 2009), Barcelona, Spain, April 2009. 